\title{
Designing collaborative blockchained signature-based intrusion detection in loT environments
}

\section{Li, Wenjuan; Tug, Steven; Meng, Weizhi; Wang, Yu}

\section{Published in:}

Future Generation Computer Systems

Link to article, DOI:

10.1016/j.future.2019.02.064

Publication date:

2019

Document Version

Peer reviewed version

Link back to DTU Orbit

Citation (APA):

Li, W., Tug, S., Meng, W., \& Wang, Y. (2019). Designing collaborative blockchained signature-based intrusion detection in loT environments. Future Generation Computer Systems, 96, 481-489.

https://doi.org/10.1016/j.future.2019.02.064

\section{General rights}

Copyright and moral rights for the publications made accessible in the public portal are retained by the authors and/or other copyright owners and it is a condition of accessing publications that users recognise and abide by the legal requirements associated with these rights.

- Users may download and print one copy of any publication from the public portal for the purpose of private study or research.

- You may not further distribute the material or use it for any profit-making activity or commercial gain

- You may freely distribute the URL identifying the publication in the public portal 


\section{Accepted Manuscript}

Designing collaborative blockchained signature-based intrusion detection

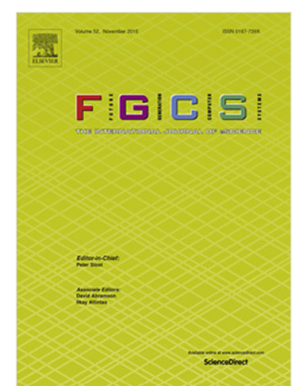

in IoT environments

Wenjuan Li, Steven Tug, Weizhi Meng, Yu Wang

PII:

S0167-739X(18)32723-7

DOI:

https://doi.org/10.1016/j.future.2019.02.064

Reference:

FUTURE 4817

To appear in: $\quad$ Future Generation Computer Systems

Received date: 31 October 2018

Revised date: 15 January 2019

Accepted date: 22 February 2019

Please cite this article as: W. Li, S. Tug, W. Meng et al., Designing collaborative blockchained signature-based intrusion detection in IoT environments, Future Generation Computer Systems (2019), https://doi.org/10.1016/j.future.2019.02.064

This is a PDF file of an unedited manuscript that has been accepted for publication. As a service to our customers we are providing this early version of the manuscript. The manuscript will undergo copyediting, typesetting, and review of the resulting proof before it is published in its final form. Please note that during the production process errors may be discovered which could affect the content, and all legal disclaimers that apply to the journal pertain. 


\title{
Designing Collaborative Blockchained Signature-based Intrusi,- Detection in IoT environments ${ }^{\text {th }}$
}

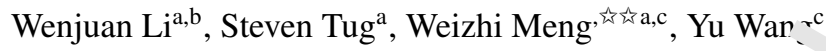 \\ ${ }^{a}$ Department of Applied Mathematics and Computer Science, Technical University , $\mathrm{L}$ nmark, . Jenmark

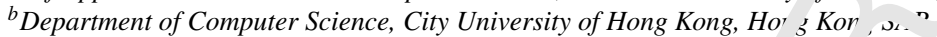 \\ ${ }^{c}$ School of Computer Science, Guangzhou University, Chiı. \\ ${ }^{d}$ E-mail address: weme@dtu.dk
}

\begin{abstract}
With the rapid development of Internet-of-Things (IoT), there is an inc. asitı demand for securing the IoT environments. For such purpose, intrusion detection systems (IDSs) are one of $\mathrm{t}_{1}$ e most important security mechanisms, which can help defend computer networks including IoT against val. 's th eats. In order to achieve better detection performance, collaborative intrusion detection systems or networks ' CIDSs or CIDNs) are often adopted in a practical scenario, allowing a set of IDS nodes to exchange requir ${ }^{\lambda} \ldots r \ldots . .$. on with each other, e.g., alarms, signatures. However, due to the distributed nature, such kind of collaboratıv network is vulnerable to insider attacks, i.e., malicious nodes can generate untruthful signatures and share and greatly degrade the effectiveness of IDSs. With the ad $n t$ of blockchain technology, it provides a way to verify shared signatures (rules). In this work, our motivation to de elop CBSigIDS, a generic framework of collaborative blockchained signature-based IDSs, which can incremeı ' 11 , huild and update a trusted signature database in a collaborative IoT environment. CBSigIDS can provid - verit ble manner in distributed architectures without the need of a trusted intermediary. In the evaluation, our resuı. demonstrate that CBSigIDS can enhance the robustness and effectiveness of signature-based IDSs under adversarial scenarios.
\end{abstract}

Keywords: Intrusion Detection System, Inte net-of- ^ 'ings, Signature-based Detection, Collaborative Network, Blockchain Technology, Insider Attacks.

\section{Introduction}

The Internet-of-Things (IoT) re elı ${ }^{+}$) a system of internet-enabled computing devices, mec.anical and digital machines, and objects th $i$ ha e the capability to transfer data over a network $\mathrm{w}^{\prime}$ ' ${ }^{\mathrm{O}} \mathrm{O}$ ' $i$ requiring humanto-human or human-to-comr iter in « action [14]. More and more organizations ar usi $\mathrm{g}$ Io $\mathrm{T}$ to improve their performance, i.e., operating - re $f$ riciently, better understanding, improving acision-ı. aking, etc. While the interrelated IoT devic s are als threatened by many attacks, i.e., the threat-tr $\wedge$ sta' s moving from manipulating informatior ، controlling actuations [2].

To safeguard arious oT devices and critical infrastructures, intru in etection systems (IDSs) are

\footnotetext{
A preliminary er on of this paper appears in Proc. of the 1st IEEE International C、 ference on Blockchain (IEEE Blockchain), pp. 1228-1235, 2018 [1].

Corresponding author: Email - weme@dtu.dk, phone and fax: $+4545253068$
}

Preprint submitted to Elsevier one of the most essential and important tools that can help identify potential anomalies and policy violations [37, 42]. Based on the deployment, an IDS can be classified as either host-based IDS (HIDS) that focuses on local system logs, or network-based IDS (NIDS) that monitors network state and traffic. Further, there are two typical detection approaches: signature-based detection and anomaly-based detection. The former like [50, 40] (also known as misuse detection) uses a signature matching process to compare the stored signatures and the observed events like payload and system record. The latter like $[49,12]$ identifies a potential threat by discovering a significant deviation between its pre-defined normal profile and the observed events for a period of time. If any security violations are found, an alarm would be sent to notify security administrators. Figure 1 depicts the high-level detection workflow of both signature-based and anomaly-based approach.

With the rapid development of cyber attacks, it has 


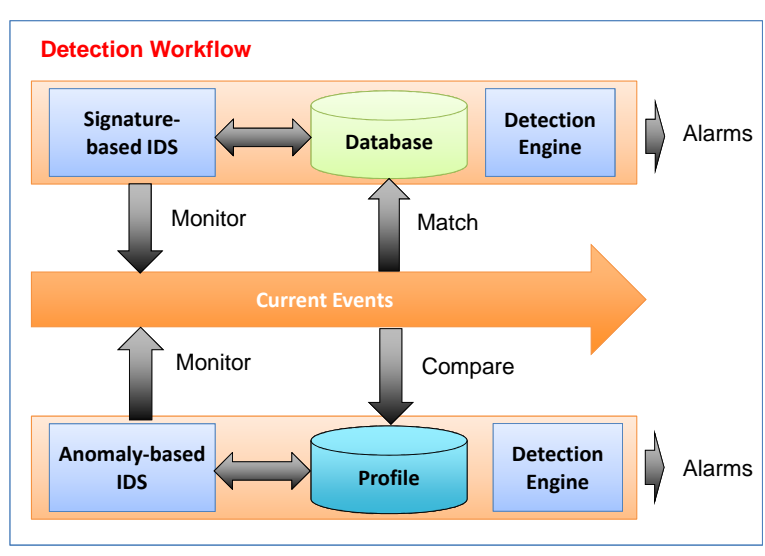

Figure 1: The high-level workflow for both signature-based and anomaly-based detection.

become much difficult for separated IDSs to accurately discover complicated attacks, as they only have limited information on the protected environments. Therefore, traditional IDSs could be easily bypassed by both wellprepared attackers and complex attacks, e.g., Denial-ofService (DoS) attack. To enhance the detection per formance in practice, collaborative intrusion detection systems or networks (CIDSs or CIDNs) are emploved, which encourages a set of IDS nodes to request and . trieve data from other nodes [53]. As an example, ID$\mathrm{S}$ nodes can share their signatures (also nam $\ldots$. $\mathrm{les}$ ) with others in a CIDN, with the purpose i impro ing detection accuracy and reducing unwante alar .1s $[25,30,24]$. However, such type of cr labr ative intrusion detection is usually vulnerable tc 'nsi er at acks, due to the distributed nature, i.e., $m r$ iciou $n$, es can provide false rules to affect the $\mathrm{de}^{t}$, inn performance of other nodes. In this case, there is a grea seed for designing appropriate security me na sms to secure the process of signature sharing in $\mathrm{CI}^{\mathrm{T}} \mathrm{S} / \mathrm{CIDN}$.

Motivations. Inspired b a broa' adoption of Bitcoin, blockchain technolo, $\mathrm{y} \mathrm{h}^{\prime} \mathrm{s}$ attacted much more attention from both academia nd ir dustry, allowing untrusted individuals to onnect wich others in a verifiable manner without he need of a trusted centralized entity [55]. A blockch. in is .n ordered list of block$\mathrm{s}$, in which each $\mathrm{st}$ them has a cryptographic pointer to their precurso New b cks can be appended to the blockchain using a $>n$ n asus protocol, which eventually allows : ou f hlockchain nodes to synchronize their copies of , ${ }^{1} \mathrm{r}$ kchain locally. By taking advantage of consensus me 'anisms, blockchains can provide a transparent and integrity protected data storage, whereas the recorded data in any given block cannot be mod- ified retroactively without the $\mathrm{mr}$ dification of all subsequent blocks. This characterist' $\iota$ 'hlockchains is desirable for sharing IDS signature in a secu . : way for CIDN and IoT environments.

Contributions. Motiva $\mathrm{d} b$ the recent development and applications of bloc hain, in this work, we focus on signature-based IDCs anc Jesign $C B S i g I D S$, which is a generic blockch in-t ad framework for securing signature sharing $a_{0}{ }^{i n}$. malicious nodes in IoT environments. The ker ide' ahind is to apply blockchain technology for ncrem tally building a trusted signature database. This can ensure the detection effectiveness by ado $\cdot$ ing on, rusted and verified signatures in a collabor tive $v_{1}$ network. Our contributions can be summarizeu us foll ws:

- To reduc the influence of malicious nodes, we p. nose : blockchain-based framework called $C B$ roIL, oy combining blockchains with distributed cirn ure-based IDSs in an IoT environment. Our approach enables various IDS nodes to incrementai y generate and verify a signature database in CIDNs. With the use of blockchains, CBSigIDS can provide a verifiable manner for sharing signatures among different nodes without the need of a trusted intermediary.

- In the evaluation, we study the performance of $C B$ SigIDS in different environments and adversarial scenarios, e.g., in a simulated and a real CIDN environment respectively. We further compare our approach with a blockchain-based SDN application called DistBlockNet [43] in a practical IoT environment. The obtained results demonstrate that CBSigIDS can enhance the robustness and effectiveness of signature sharing in a CIDN through building a trusted signature database, i.e., it can protect DistBlockNet against malicious nodes.

It is worth noting that this work focuses mainly on signature-based detection, which has a larger implementation in practice as compared with anomaly detection [46]. This is because anomaly-based IDSs often result in a high false alarm rate due to the difficulty of building an accurate profile. While the combination of blockchain technique and anomaly detection is one of our future work. The major purpose of this work is to explore the feasibility of applying blockchain technology in CIDNs, and to stimulate more research in designing robust signature sharing in CIDNs.

The remainder of this paper is organized as follows. Section 2 introduces related research studies in relation to distributed and collaborative intrusion detection. In 
Section 3, we introduce the background of blockchain technology and describe CBSigIDS in detail, e.g., the high-level architecture on how participating nodes construct a consortium blockchain. Section 4 presents our evaluation settings and discusses the obtained results. Section 5 presents some limitations and challenges in this field. Finally, we conclude our work in Section 6.

\section{Related Work}

Traditionally, a separated IDS often has no information about the deployed network where it tries to protect, leaving an opportunity for attackers to bypass its examination. For instance, cyber intruders can launch some complex attacks like DoS attack to compromise a single IDS, as it cannot have an overview of the whole traffic status in a network. In this case, there is a great need for a collaborative system or IDS network to leverage the detection performance of a single IDS [53].

Distributed systems. In the literature, distributed monitoring systems have been developed for decades. For example, distributed Intrusion Detection System (DID $S$ ) [44] was introduced in 1991, which could utilize distributed monitoring and data reduction with centra ${ }^{1} \cdot \operatorname{cod}$ data analysis module to analyze a heterogeneous con. puter network. Event Monitoring Enabling Responses to Anomalous Live Disturbances (EMERALD' 'ני, 'as developed in 1997, which could track malici us activi y across abstract layers in a large network. It cu. hi' es models from distributed high-volume ev' ats r ith traditional intrusion detection. COSSACK $s^{\text {te }} \mathrm{n}\left[3^{r}\right]$ was designed for mitigating DDoS attac'. in an 'omatic way. This system does not require . u. 'an intervention and supports independent attack signature $z$ neration.

In addition, DOMINO (Distri' uter Overlay for Monitoring InterNet Outbreaks) $\left[\gamma^{7} v\right.$ as another type of distributed IDS, which enh ices c " ${ }^{1}$ aboration among heterogeneous nodes in a' atwr ' $\mathrm{k}$. The overlay design enables this system to be ho oge eous, scalable, and robust to attacks and fa; ues. It rius the capability of detecting spoofed IP sou ces, red ، ing false positives, and classifying threats in a ' mely ıanner. Then, PIER [13] was an Internet- ale query engine, which supports massively distr suted, d tabase-style dataflows for snapshot and contiı nus $r$ teries. It can serve as a building block for af diverse Internet-scale informationcentric applicaı irs.

Collaborative intrusion detection. In order to achieve better detection performance, a CIDS or CIDN enables an IDS node to exchange require information with other nodes. In 2006, Li et al. [15] y. - -ed out that most distributed IDSs were relying on either cu tralized fusion, or distributed fusion that ar, no unscalable. Motivated by this issue, they propose - t v se of CIDS based on the emerging decentralized - cation. and routing infrastructure. However, their mochaiı $\eta$ assumes that all peers in the network are $t$ sste which would be vulnerable to insider attacks. i. th $f$ field of collaborative intrusion detection, insider ${ }^{n}{ }^{+t a c k}$ are considered as one of the biggest threats. where .7 intruder has the right to consume resources $\eta$ a netr ork.

To protec ${ }^{+}$IID/v usainst insider threats, a promising solution is to $\mathrm{d}^{\mathrm{s}}, \mathrm{g}$. appropriate trust mechanisms to evaluate the . putat on levels among IDS nodes. As an example, \& 'ma et al. [5] introduced a P2P-based overlay method for IDSs (shortly Overlay IDS), which uses a trist-a are ngine for correlating alerts and an adaptive sc. sme for managing trust. Tuan [48] proposed an r r w on using game theory to model and analyze the pru sses of reporting and exclusion in a P2P network. Thav concluded that if a reputation system was not in$c^{\prime}$ ntive compatible, the more numbers of peers in the $s$ s stem, the less likely that a malicious will be reported orrectly.

Based on this observation, Fung et al. [8] proposed a challenge-based CIDN, where the trustworthiness of an IDS node depends on the received answers to the challenges. They first introduced a Host-based IDS framework that enables each HIDS to evaluate the trustworthiness of others based on its own experience and uses a forgetting factor to give more emphasis on the recent experience of each peer. To improve the performance of such mechanism, Li et al. [16] identified that differen$\mathrm{t}$ IDS nodes may have different levels of sensitivity in detecting different types of intrusions. They then proposed a notion of intrusion sensitivity (IS) that measures the detection sensitivity of an IDS in detecting different kinds of intrusions. Accordingly, they proposed an intrusion sensitivity-based trust management model [17] that could allocate the values of $I S$ by means of machine learning classifiers (e.g., a knowledge-based KNN classifier [30], ensemble classifier [25]). As a study, they described how to apply intrusion sensitivity for alarm aggregation and investigated its effect on defeating pollution attacks, in which a group of malicious peers cooperate together by providing false alert rankings [19]. The experimental results indicated that their method can decrease the trust values of malicious nodes in a fast manner.

Li et al. [20, 22] further identified intruders could use some advanced attacks to compromise a challenge 
mechanism. They introduced a passive message fingerprint attack (PMFA), which enable malicious nodes sending malicious feedback to only normal request and their trust values. They also developed a special OnOff attack (called SOOA) [23], in which malicious nodes could keep responding normally to one node while acting abnormally to another node. In addition, how to reduce the overload in communication is a critical issue for challenge mechanisms in different scenarios, e.g., healthcare $[21,34]$. Some other related work regarding how to enhance the performance of an IDS can be referred to $[6,7,18,26,27,28,29,31,32,35]$

Blockchain-based intrusion detection. How to apply blockchains in the field of intrusion detection is an interesting and important topic. Many studies have started researching in this area. Alexopoulos et al. [3] described a framework of a blockchain-based CIDS, where they considered a set of raw alarms produced by each IDS as transactions in a blockchain. Then, all collaborating nodes employed a consensus protocol to ensure the validity of the transactions before delivering them in a block. This can guarantee the stored alerts are tamper resistant in the blockchain, but they did nc implement and evaluate their method in practice.

Focused on this issue, Meng et al. [33] pror :-d some early insights regarding the intersection of IDS. and blockchains, and discussed some challenges in this area. They believed that blockchains can ha $\approx$ a $r$ sitive impact on distributed intrusion detectic in the a pects of data sharing, alarm exchange ar $\mathrm{d}$ trus. $\mathrm{C}_{\mathrm{C}} \mathrm{n}$ putation. Golomb et al. [11] then intror iced CIoTA, a framework that uses the blockchain cor or . to ' erfor$\mathrm{m}$ distributed and collaborative ano' raly de those devices with limited resourcer . ᄂ. the other hand, IDS technique can also help protect blockviain applications. Steichen et al. [47] prr post 1 ChainGuard, an OpenFlow-based firewall for st ri $^{\prime} \mathrm{g}$ blockchain-based SDN, which requires all tr fffic to 'e blockchain nodes should be forwarded ' $y$ th swi $^{+}$ches controlled by ChainGuard. This could he $\mathbf{e}_{\boldsymbol{}_{\mathbf{r}}}$ edu e the malicious behavior from the partici aung nous. Sharma et al. [43] proposed DistBlockNe, a distrı uted secure SDN architecture for IoT by intes ating ne blockchain technology, allowing a no e to irteract with others without the need of a trusted entral ci ntroller.

How to share rus - in - secure way is an important issue in the fie . . . Ournsion detection [9]. Our previous work [1] introw $c d$ how to achieve this by leveraging blockchain techn' 'ygy. In this work, we further consider DistBlockNet in the evaluation, and evaluated both CBSigIDS and DistBlockNet in a practical IoT environ- ment. It is found that CBSigIDS an be used to enhance the robustness of DistBlockNet a $\therefore$ fending against malicious nodes.

\section{Our Approach}

In this section, w $w$, jin by introducing the background of blockch in te nnu.ogy, and then introduce how to design CBSigie : for CIDNs in detail.

\subsection{Blockchai, Techno, gy}

Blockch? ... can u considered as a distributed data structure, llov .ng information to be shared and verified amnng uffere $i$ entities in a peer-to-peer network, without thu veea of a trusted third party. In other words, bloc $^{1}$ chain tec nology is a decentralized ledger that enablas re " $\mathrm{dir}$ ó transactions across various participating nodes, nd protecting data integrity via strong cryptogsing wurs. The recorded data in any given block cannor a altered retroactively without the alteration of all

hcenuent blocks $[33,54]$. A typical blockchain contr ns a list of records (called blocks) that can be chronoh rically ordered by discrete time-stamps. In particular, - ?ch block is linked to the previous block via a cryptographic hash, and the first one is called genesis block. A block usually contains a payload, a time-stamp and a cryptographic hash value of the entire previous blocks in the chain. Thus, blockchains can be treated as an implementation of a shared secure distributed ledger, where the participants have the right to read and write without any constrains in most cases.

According to specific types of permission control, existing approaches of blockchain implementation can be categorized into three types: public, consortium, and private. More specifically, a public blockchain allows every entity to act as a reader and a writer without any constrains, like Bitcoin [36] and Ethereum [52]. A consortium blockchain only allows registered entities or a small group of verified entities to have the right to read or write on the blockchain. A private blockchain is often controlled by a single entity, but still can be distributed in different locations. A blockchain can be updated via a consensus protocol, which ensures all participating entities to agree on a uniform view of the ledger. A consensus protocol can be dependent on specific blockchain implementation and threat model [33].

- Proof of work. This method allows a node to successfully accept a block, when a pre-defined amount of computational resources (known as 'work') can be proved to spent. 


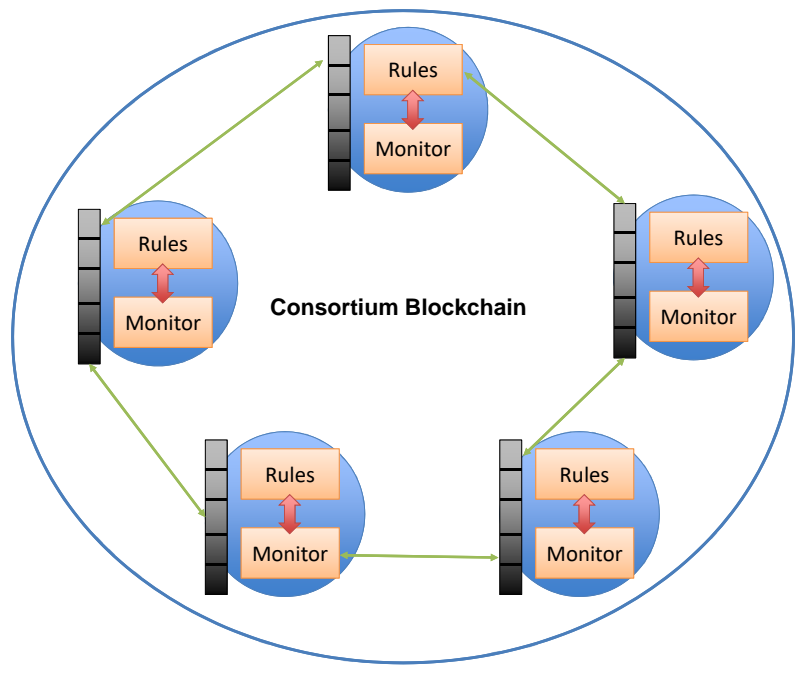

Figure 2: The high-level architecture of CBSigIDS, where the participating nodes can construct a consortium blockchain.

- Proof of stake. This method ensures a consensus to be achieved by considering both random selection and the influence (known as 'stake') of the participating entities. It is assumed that entities woul guarantee the integrity of blocks when they have a large stake in the blockchained network.

- Proof of elapsed time. This method ensures a consensus to be achieved by requesting every pu tial verifier to share a secure and random $v$ iting tin 2 from a trusted execution environment

\subsection{CBSigIDS}

As discussed earlier, collaborativ , intrusı. detection encourages IDS nodes to shar ; 1 . 'ired information with each other in order to enhance thi detection capability. For example, a sign r ure- ased IDS can update its own rule database and an share some rules to help other nodes improve th $r$ detec $\_$' $\eta$ performance in a network. However, we $\mathrm{n}$, tice nat CIDNs are typically vulnerable to various insic attr $\mathrm{ks}$; thus, an insider can share false signatur s to degraue the effectiveness of detection, i.e., hiding xternal : tackers.

Motivated by the wh adn ion of blockchain technology, in this $\mathrm{w}, \mathrm{rk}$, we focus on insider threats and propose CBSigIl S, whic is a generic framework of collaborative bloc hair $\mathrm{d}$ signature-based IDSs. It mainly leveri $:-1$ r chains to help build a trusted rule database in a c. 11 . oorative network environment. Figure 2 depicts the igh-level architecture of CBSigIDS, in which the participating nodes can construct a consortium blockchain.
It is worth noting that consorti $\mathrm{m}$ blockchains are applicable in existing CIDNs, wh in vnly a group of verified nodes can join and interact with - ich other in the network. For instance, cha' ens -based CIDNs require a node to register to a trus. $d r$ rtificate authority $(C A)$ and obtain its unique $\mathrm{p}$. 'of $\mathrm{o}_{\mathbf{1}}$ 'rentity (e.g., a public key and a private key) hefor it can join a CIDN. This attempts to provide ; rirst $\cdots$ ref of defence against malicious nodes, i.e., av $\mathrm{Air}_{\mathrm{g}}$ a participant to register many identities.

As depicted in Figu. 2, a signature-based IDS node often conta' 's thre' major components, including $\mathrm{P} 2 \mathrm{P}$ comm' ... ation component, collaboration component and $t$ ist $r$ anc rement component $[8,16,17]$.

- P2P cor. nunication. This component is responsi$\therefore$ for es $t$ blishing a connection with other IDS n-des susarding network organization, management ana 'ssibly physical communication.

- Cullaboration component. This component is used to allow an IDS node to collect required information to evaluate the trustworthiness of target nodes, and send corresponding feedback requested by other nodes.

- Trust management component. This componen$\mathrm{t}$ is responsible for implementing trust computation and evaluating the reputation levels of target IDS nodes. As an example, challenge-based trust mechanism investigates the reputation of a node by comparing the received feedback with the expected answers [8, 17].

Threat model. In this work, we assume that an attacker can control one or several nodes in a CIDN, but cannot successfully manage a large number of IDS nodes within a short period of time. In addition, as each CIDN node has a pair of private and public key, their identities cannot be easily manipulated and duplicated.

CBSigIDS blockchain. In CBSigIDS, each IDS node (or blockchain node) in the consortium blockchain can monitor the network traffic, identify attacks and periodically share a set of signatures (rules) with others. This set of rules has to be signed by a private key from a node, in order to understand the source of rules. Other nodes will only accept these rules by verifying them against their local database. In this case, the blockchain can be only expanded if the majority of nodes have verified that the received block contains trusted rules. 


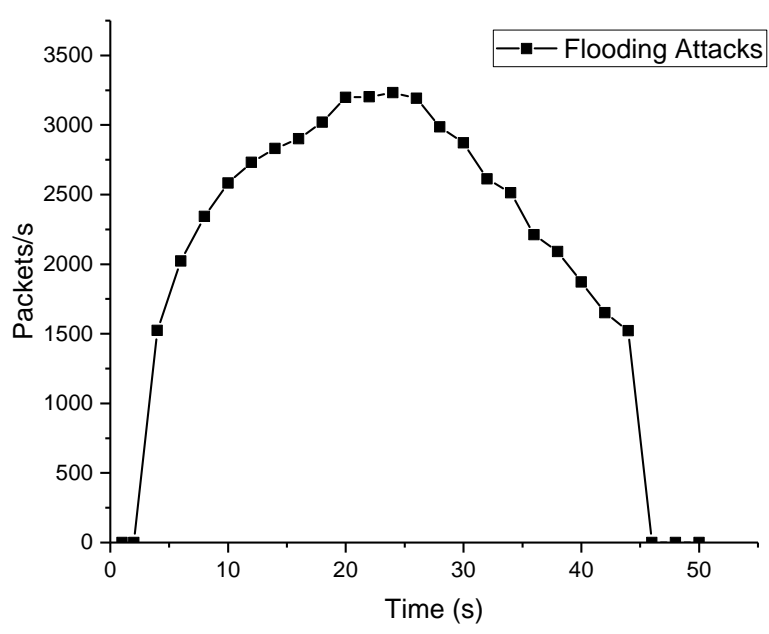

Figure 3: The packet rate during the period of flooding attack.

\section{Evaluation}

In this section, we evaluate the performance of CBSigIDS under some adversarial scenarios in a simulated and a real CIDN environment, respectively.

\subsection{Experiment-1}

In this experiment, our goal is to investigate the $r$ "formance of CBSigIDS against worm attack and flooding attack in a simulated CIDN. Our simulated " "xork contains a total of 50 nodes that were ran' omly a. tributed in a $10 \times 10$ grid region. We used $\mathrm{s}$. ort $[4$, as the signature-based IDS and adopted is default . ale database. The experiment could be start $\mathrm{d} \mathrm{w}^{\prime}$ en a'. IDS nodes built a list of neighbors and es dblis. $-d$ stable connection. When an IDS node upd is its rules, it can share the rules with others via the ilockc. in.

Flooding attack. To test to $\approx \mathrm{p} \in$ formance of $C B$ SigIDS, we randomly selectea "c outside nodes (not belong to our CIDN) to laur h a flo 'ing attack, while an IDS node inside the CII $\mathrm{N}^{-{ }^{*}}$,ted haring two related rules against such attack. $5_{1_{c}}$ re ? presents the packet rate during the floodir, pericd. ihe attack was started from $4 \mathrm{~s}$ and stopp 1 at $44 \mathrm{~s}$, in which the maximum packet rate could reach . "nun' 3200 pacekts/s.

Figure 4 depict the number of infected nodes during the flooding attac $\therefore$. 'Infec ed' nodes here refer to those nodes who failed 4 nrn . pt an alarm for the launched flooding atta . - norally, if a blockchain node accept$\mathrm{s}$ the shared $\mathrm{ru}_{\mathbf{1}} \mathrm{s}$ it has the capability of detecting the flooding attack. $C$ r obtained results indicated that $C B$ SigIDS could help steadily decrease the number of infected nodes, i.e., from an initial number of 49 to 10

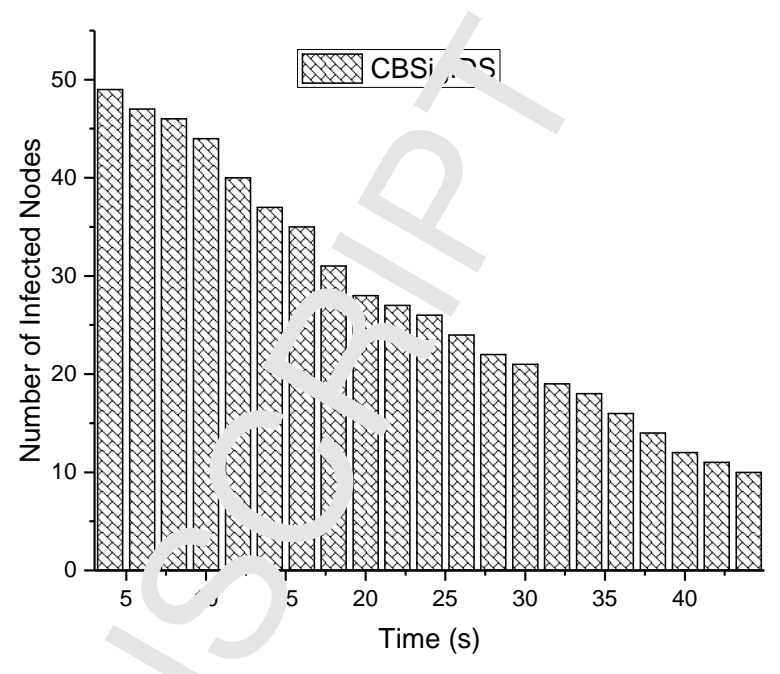

Figuı 1. The nu lber of infected nodes during the flooding attack.

$a_{n}$ ing the flooding attack. We also found that the decreas11. speed depends heavily on the verification and ur daung procedure in the blockchain.

Worm attack. Under this attack, it is assumed that three IDS nodes updated its rules and started sharing related rules with others via the blockchain, and that the other nodes were not capable of detecting the worm at that time (denote as vulnerable node). Only the vulnerable nodes those who accepted the rules before being hit by the worm, could immediately protect themselves (denote as survived node) and mitigate such attack by reacting in a proper way, i.e., closing the vulnerable port or disconnecting from the network until the vulnerability is fixed. During the attack period, worm would be distributed to IDS nodes in every 2 seconds.

Figure 5 depicts the number of survived nodes under the worm attack. It is found CBSigIDS could gradually increase the number of survived nodes to 32 , with a survival rate of $66.7 \%$. In [5], they used a P2P-based overlay for intrusion detection that addresses the wor$m$ attack using both a trust-aware engine for correlating alerts and an adaptive scheme for managing trust. They evaluated their method with a virtual network with 36 clients and an Internet worm attack. The overlay IDS can produce an alarm if it receives three similar alert messages from other nodes. In our experiment, our work could achieve a similar but better result (66.7\%), as compared with the overlay IDS (a survival rate of $60 \%$ ). These results demonstrate that the feasibility and performance of our approach in securing the CIDNs under attacks. 


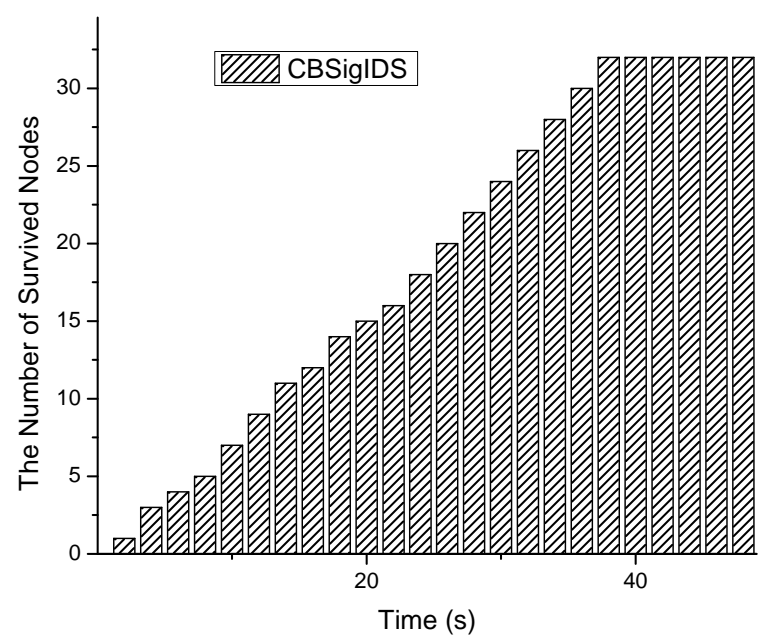

Figure 5: The number of survived nodes under the worm attack.

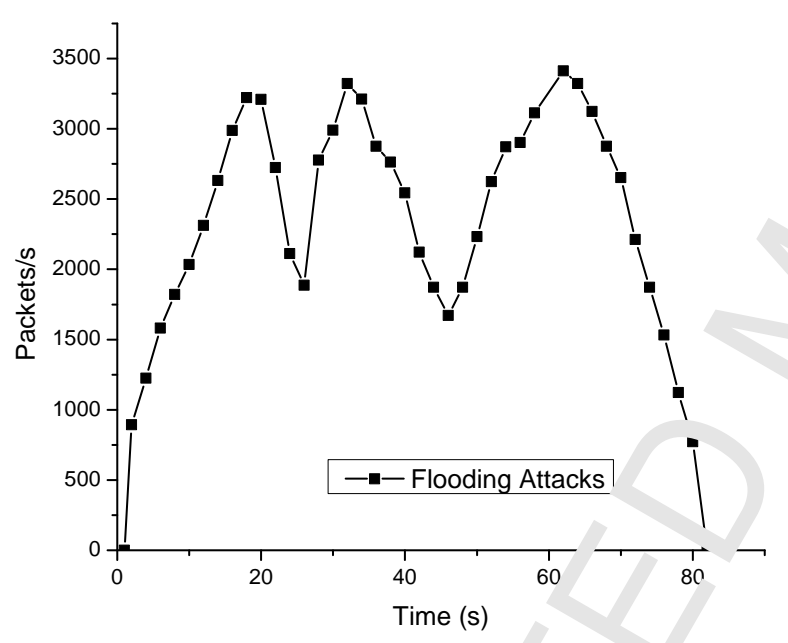

Figure 6: The packet rate during the period', flo ' " $n g$ attack in a real CIDN environment.

\subsection{Experiment-2}

In this experiment, we $\mathrm{c}^{\prime}$ llab' rated with an IT company to study the perforı. $n c$ of BSigIDS in a real CIDN with a total of 46 nodes. " ne IDS nodes could connect with the Inter et thro rh a server that could also provide many comp iting re ources. We implemented our approach $\cdots+$ a p.uof-of-concept blockchain, and Snort [45] w s deplo, ad in each node.

Flooding attack. Sim: ar to the first experiment, we also utilized w... svternal nodes to launch a flooding attack, whi $\Rightarrow$ in IDS node inside the CIDN started sharing releva. ${ }^{+}$rules to defeat such attack. Figure 6 shows that the period of attacking traffic was started from $2 \mathrm{~s}$ and stopped at $80 \mathrm{~s}$, in which the maximum

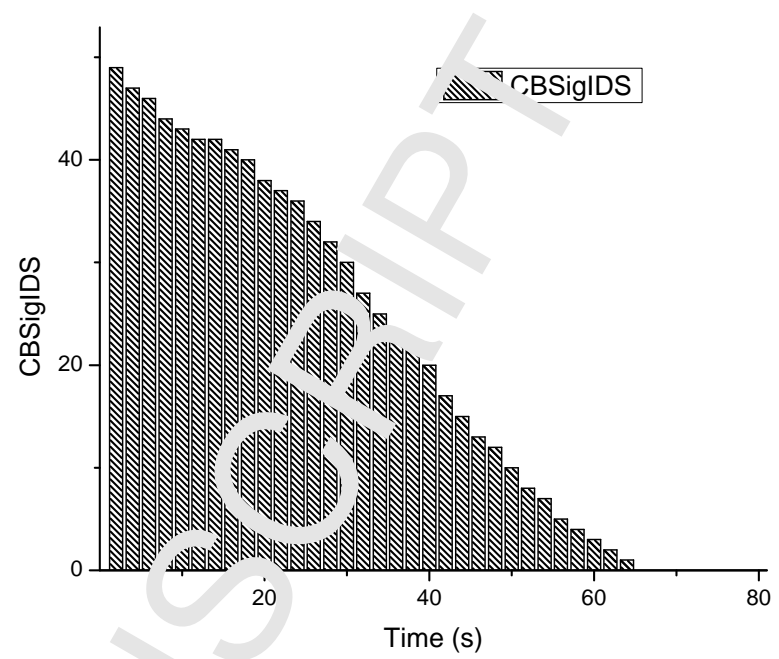

Figur 7: The nun ser of infected nodes during the flooding attack in a real ClL.

- uket rate could reach around 3450 pacekts/s. Figure 7 desc. 'hes the number of infected nodes during the peri$\therefore{ }^{c}{ }^{f}$ ooding attack. It is found that our approach could sadily reduce the number of infected nodes from 49 to 0 'means that all nodes could produce alarms for the .. joding attack), when the time reached 66s.

Insider exploration. To explore the effectiveness of signature sharing, we randomly selected one node inside the CIDN to be malicious, which could share false rules with other nodes. We mainly manipulated the patterns in these signatures (rules) that are used to detect flooding attack and worm attack. The main purpose behind this exploration is to study the impact of malicious nodes on CBSigIDS. We repeat such exploration several times, and found that these false rules would not be accepted by other nodes, as they could not bypass the verification in the blockchained signature database. Our obtained results validate that an attacker cannot compromise our approach of CBSigIDS, if he fails to manage the majority of blockchain nodes in a CIDN.

\subsection{Experiment-3}

In this experiment, we collaborated with another IT organization and established an SDN-based IoT environment. Figure 8 shows the high-level network architecture, including a controller layer and an IoT device layer. In particular, the controller layer contains three SDN controllers that could synchronize information, and device layer consists of 56 IoT devices, like PCs, laptops and smartphones, which are managed by the controllers. Snort was deployed in each node to perform signature-based detection. 


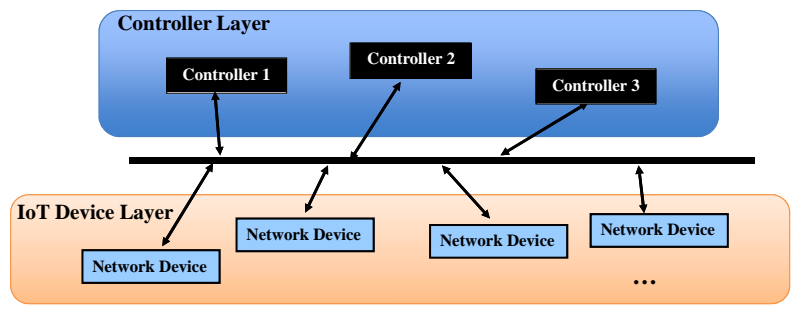

Figure 8: The high-level architecture of blockchain-based SDN.

DistBlockNet. In this experiment, we consider DistBlockNet [43], which employs distributed network control in the IoT network by using the blockchain technology to improve security, scalability, and flexibility, without the need for a central controller. In particular, they applied blockchain technique for updating a flow rule table, in order to securely verify a flow-rule table. To mitigate different types of attacks, it could deploy some additional security mechanisms for threat prevention, data protection, and access control. For example, it implemented two modules called Shelter and OrchApp in each local network to help handle the security attacks at a different level. OrchApp mainly works at the man agement or application layers, the controller-application interface, and the control layer. Shelter handles the $\ldots$ layer, the controller-data interface, and the control laye. However, we found there was no particular mechanism in the IoT device layer to identify false dat? chat 1 . 7y be sent by malicious nodes. Their evaluatio. results i dicated that DistBlockNet could detect mr icious a ic under flooding attacks.

Flooding attack. Similarly, we u ed solı xternal nodes to conduct a flooding attac' . . - $d$ deliver malicious traffic to such IoT environment. As s.. 'wn in Figure 9, the period of malicious trs nc as started from $3 \mathrm{~s}$ and stopped at $100 \mathrm{~s}$, in which : a $\mathrm{m}$.ximum packet rate could reach around $3772 \mathrm{par}$ kts/s.

Different from the abov, twr exp-riments, this time we assume that all insider nc's ho e one effective rule in detecting this attack and set $u_{r}$ two malicious nodes to started spreading $\mathrm{n}$ alicious ules that attempt to replace the effective rule rith - ralse one from $3 \mathrm{~s}$. Figure 10 depicts the number of infected nodes during the period of floodin r attack. 'Infected' here refers to the nodes who adopt th. fals rule during the attacking period. It is foun a.... nictBlockNet was vulnerable to such attack, where tı. ; ifected nodes could increase gradually and all nodes b. `ome infected at around 80s. This is because DistBlockNet did not employ a particular mechanism to check the signatures sent by insider nodes.

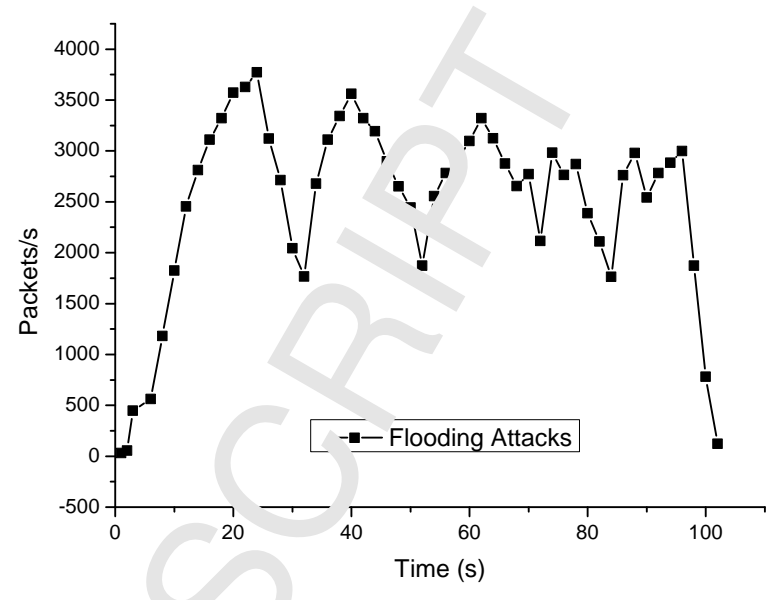

Figure 9: 1. nacku rate during the period of flooding attack in the IoT environment.

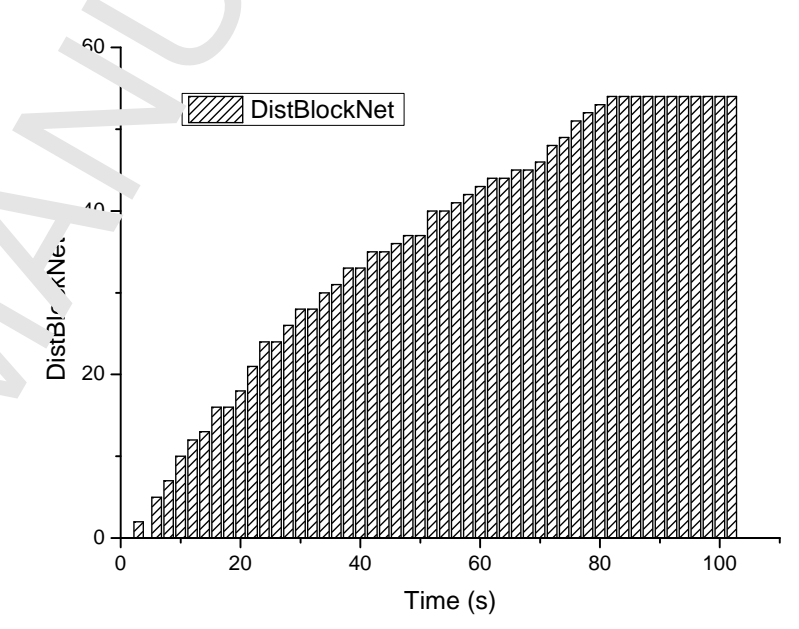

Figure 10: The number of infected nodes during the flooding attack in the IoT environment

As a comparison, we applied our approach into DistBlockNet and re-performed the experiment three times. We found that the false rule would not be accepted by insider nodes under CBSigIDS, as the false one could not bypass the verification of our blockchained rule database. Our results demonstrate that our approach of CBSigIDS can help enhance the robustness of a collaborative signature-based CIDN by building a trusted signature database.

\section{Discussion and Limitations}

To the best of our knowledge, this is the first work in discussing the application of blockchains with collaborative signature-based IDSs. There are many issues and open challenges in this emerging area. 
- Signature-based detection. As signature-based IDSs often produce fewer false alarms than an anomaly-based IDS, it has been more extensively used in practice [46]. Therefore, this work mainly focuses on signature-based IDSs and explores how to share rules in a verifiable way. It is worth noting that Golomb et al. [11] has tried to combine blockchains with anomaly-based detection through building a trusted training model. Based on the results obtained in this work, it is one of our future topics to consider how to build a more effective and robust collaborative anomaly-based IDS.

- CIDN environmens and CBSigIDS blockchain. In this work, we explored the performance of $C B$ SigIDS in a simulated and a practical CIDN, respectively. In practice, CIDNs have been widely implemented, whereas blockchains are still under construction especially in the field of intrusion detection. As a result, we only adopted a proofof-concept blockchain in current work. In future work, we plan to validate our approach using more practical and well-developed blockchains.

- Adversarial scenarios. Similar to other studies in the area of intrusion detection, this work $\mathrm{m}^{\cdots}$. considers several common attacks like flooding at tack and worm attack. The obtained experimental results demonstrated the feasibility and Itec $_{\mathrm{i}} \mathrm{e}-$ ness of our approach. In future work, + is an $\mathrm{i}$ teresting topic to consider other atta ks in ${ }^{\prime} \mathrm{dg}$ advanced attacks, and different netr ork ettings.

- Trust mechanisms. Currently. most L.'Ns are likely to deploy at least one tr st-. 'sed mechanis$m$ to help identify insider attacks, like challengebased mechanism that ev uat $s$ the trustworthiness of target nodes by $-m$, aring the received feedback with the exr cted a. vers. In future work, we plan to cond act a jomrarison among several trust mechanisms $w^{\prime}$ a bl ckchain technology in securing CIDN _nvironme.ıts.

- Large-scale evaluc inn. io investigate the scalability of a ser arity mechanism. It is very importan$t$ to perform a large nd systematic evaluation by considering $\mathrm{V}_{a}$ : $_{\mathrm{nc}}$ ariables and scenarios. However, bl ... : in -hased intrusion detection is an emerging th $i$, some special conditions should be considered $\mathrm{o}$. how to design such kinds of experiments, i.e., which type of blockchains can be used in the evaluation.
Intuitively, blockchain techno jgy can help improve intrusion detection in the asp $i$ of data sharing and alarm exchange, but it still suffers frus.. some inherent challenges and limitations : $:$ cor ling to [33].

- Energy and cost. The c mputational power is a concern for blockcha1ı 'nplications in real-world scenarios. For : ista 1 re, Wang and Liu [51] identified that the 'qui ed computational power could be added on sing $\mathrm{n}$ niners at first, while could be greatly ins eased fterwards when the network evolved.

- Secur y and nrivacy. Most existing blockchain applicai on requ re smart transactions and contracts to a link ${ }^{\circ}$.o known identities. This could increase $\iota$ th privacy and security concerns when s- ring the data on the shared ledger. In addition, hlock_..uln technology can be threatened / hacked by .. ' ny traditional attacks like distributed denialof-service (DDoS) attacks.

$I$ atency and complexity. Depending on different scenarios and architectures, blockchain applications possibly require several hours to finish until all parties update their corresponding ledgers, which may open a hole for cyber-criminals. In this work, we only used a proof-of-concept blockchain instead of an existing blockchain, hence the achieved speed could much faster than that in a practical blockchain application. While the proof-ofconcept blockchain is still valid to investigate the robustness of our approach in terms of our goals. In future, we plan to construct a more practical blockchain and re-evaluate our results.

- Organization and block size. Due to the wide adoption of blockchain applications, many different organizations may develop their own blockchain related standards. Due to the increasing size of distributed ledgers, this may greatly degrade the performance and make the blockchains less efficient than current frameworks.

\section{Conclusion}

Collaborative intrusion detection has become an important and essential security solution to safeguard IoT environments, which allows various IDS nodes to exchange information with each other, e.g., rules. However, malicious nodes in a CIDN may generate untruthful signatures and share to others, which can greatly degrade the effectiveness and robustness of detec- 
tion. In the literature, blockchain technology is believed to provide a verifiable manner for sharing information without the need of a trusted centralized entity. Motivated by the recent blockchain applications, in this work, we focus on signature-based detection and develop CBSigIDS, a generic framework for collaborative blockchained signature-based IDSs, which adopts blockchains to help incrementally share and build a trusted signature database. In the evaluation, our experimental results in both simulated and real IoT environments demonstrate that $C B S i g I D S$ can enhance the robustness and effectiveness of signature-based detection under adversarial scenarios (e.g., flooding attacks) by sharing the signatures in a verifiable way.

Our work is an early research study in this area, showing how to use blockchains to improve the effectiveness of collaborative signature-based IDSs. The main purpose is to complement the literature and stimulate more research on this topic. Future work includes building a secure IDS framework via blockchains for anomalybased detection and developing a strong mechanism in defending IDS nodes against advanced insider attacks.

\section{Acknowledgment}

The authors would like to thank security adminıs trators and managers from the participating organization for their help and support during the e dluai 'n. This work was partially supported by H20? I-SU-IC :03-2018: CyberSec4Europe and the Yor ig Sc. nt' sts Fund of the National Natural Science Fou dation of China (No. 61802077).

\section{Reference}

[1] S. Tug, W. Meng, and Y. Wang, " $\mathrm{C}^{\sim}$ : $\sigma$ IDS: Towards Collaborative Blockchained Signature-b sed I rusion Detection," In: Proc. The 1st IEEE Internatic. ${ }^{-1}$ Cr iference on Blockchain (Blockchain), pp. 1228-1235 2018

[2] M. Ammar, G. Russello, an B. C ispo, .nternet of Things: A survey on the security of I $\Gamma$ fra ewor's," Journal of Information Security Applications sc n. 8-' / (2018)

[3] N. Alexopoulos, E. ' omanu. is, N.R. Ivanko, and M. Muhlhauser, "Towar ss blockı ain-based collaborative intrusion detection syster s," In: Pl c. Int. Conf. Critical Inf. Infrastruct. Secur., pp. 1- ? $70 \%$

[4] J. Douceur, "T. ث sybil attack," In: Druschel, P., Kaashoek, M.F., Rowstr 1, A. (ea ) IPTPS 2002. LNCS, vol. 2429. Springer, Heid herg, $20 \mathrm{r}$.

[5] C. Duma, M. Kaı.__. N, N. Shahmehri, and G. Caronni, "A Trust-Aw c $c, \ldots$ nned Overlay for Intrusion Detection," In DEXA Worn hc , pp. 692-697, 2006.

[6] Z.M. Fadlulla. T. Taleb, A.V. Vasilakos, M. Guizani, and N. Kato, "DTR . B: combating against attacks on encrypted protocols through traffic-feature analysis," IEEE/ACM Trans. Netw., vol. 18, no. 4, pp. 1234-1247, 2010.
[7] I. Friedberg, F. Skopik, G. Settan ו, and R. Fiedler, "Combating advanced persistent threats' ' ' $m$ network event correlation to incident detection," Co..lputers Security, vol 48, pp. $35 ? 7,2015$.

[8] C.J. Fung, O. Baysal, J. Zr .ng, I Aib, and R. Boutaba, "Trust Management for Host-B. ᄀr $r$ sllaborative Intrusion Detection," In: De Turck, F Kelı. - W. Kormentzas, G. (eds.): DSOM 2008, LNCS 527っ, , \109-122, 2008.

[9] C.J. Fung, Q. Zhu, r . utaba, Basar, "SMURFEN: A system framework fo rule in ' $\circ$ o collaborative intrusion detection," In: Procee . 'os $r$ CNSM, pp. 1-6, 2011.

[10] F. Gong, Next Genera. ' n Intrusion Detection Systems (IDS). McAfee Net ork Secrurity fechnologies Group, 2003.

[11] T. Golomb, r. Mirsky, nd Y. Elovici, "CIoTA: Collaborative IoT Anomal Detectio' via Blockchain," In: Workshop on Decentrali- 'Io1 _. . . ty and Standards (DISS), 2018.

[12] A.K.' 'nosh Vanken, and F. Charron, "Detecting Anomalous ‘ $d^{\top}$ „knor a Intrusions Against Programs," In: Proc. An 'ual Comput Security Applications Conference (ACSAC), pp. 2ذ, ?67, 1998.

[13] Huebsch, 1 , Chun, B.N., Hellerstein, J.M., Loo, B.T., Mania. P., Ro zoe, T., Shenker, S., Stoica, I., Yumerefendi, A.R.: The 5 - .tecture of PIER: an Internet-Scale Query Processor. In. Droceedings of the 2005 Conference on Innovative Data y vouns Research (CIDR), pp. 28-43 (2005)

1 ' F. Javed, M. K. Afzal, M. Sharif, B.-S. Kim, "Internet of 1. .ngs (IoT) Operating Systems Support, Networking TechJlogies, Applications, and Challenges: A Comparative Review," IEEE Communications Surveys and Tutorials 20(3), pp. 2062-2100 (2018)

[15] Z. Li, Y. Chen, and A. Beach, "Towards Scalable and Robust Distributed Intrusion Alert Fusion with Good Load Balancing," In: Proceedings of the 2006 SIGCOMM workshop on Largescale attack defense (LISA), pp. 115-122, 2006.

[16] W. Li, Y. Meng, and L.F. Kwok, "Enhancing Trust Evaluation Using Intrusion Sensitivity in Collaborative Intrusion Detection Networks: Feasibility and Challenges," In: Proceedings of the 9th International Conference on Computational Intelligence and Security (CIS), pp. 518-522, 2013.

[17] W. Li, Y. Meng, and L.F. Kwok, "Design of Intrusion Sensitivity-Based Trust Management Model for Collaborative Intrusion Detection Networks," In: Proceedings of the 8th IFIP WG 11.11 International Conference on Trust Management (IFIPTM), pp. 61-76, 2014.

[18] W. Li, W. Meng, X. Luo, and L.F. Kwok, "MVPSys: Towards Practical Multi-View Based False Alarm Reduction System in Network Intrusion Detection," Computers $\mathcal{E}$ Security, vol. 60, pp. 177-192, 2016.

[19] W. Li and Y. Meng, "Enhancing Collaborative Intrusion Detection Networks Using Intrusion Sensitivity in Detecting Pollution Attacks," Information and Computer Security 24(3), 2016.

[20] W. Li, W. Meng, L.F. Kwok, and H.H.S. Ip, "PMFA: Toward Passive Message Fingerprint Attacks on Challenge-based Collaborative Intrusion Detection Networks," In: Proceedings of the 10th International Conference on Network and System Security (NSS), pp. 433-449, 2016

[21] W. Li, W. Meng, L.F. Kwok, and H.H.S. Ip, "Developing Advanced Fingerprint Attacks on Challenge-based Collaborative Intrusion Detection Networks," Cluster Computing, Springer, 2017

[22] W. Li, W. Meng, L.F. Kwok, and H.H.S. Ip, "Developing Advanced Fingerprint Attacks on Challenge-based Collaborative Intrusion Detection Networks," Cluster Computing, pp. 1-12, Springer, 2017.

[23] W. Li, W. Meng, L.F. Kwok, "Investigating the Influence of 
Special On-Off Attacks on Challenge-based Collaborative Intrusion Detection Networks," Future Internet, vol. 10, no. 1, pp. 1-16, 2018.

[24] W. Li, W. Meng, C. Su, and L.F. Kwok, "False Alarm Reduction using Fuzzy If-Then Rules for Medical Cyber Physical Systems," IEEE Access, vol. 6, no. 1, pp. 6530-6539, IEEE, 2018.

[25] Y. Meng and L.F. Kwok, "Enhancing False Alarm Reduction Using Voted Ensemble Selection in Intrusion Detection," International Journal of Computational Intelligence Systems, vol. 6 , no. 4, pp. 626-638, 2013

[26] Y. Meng and L.F. Kwok, “ Adaptive Non-Critical Alarm Reduction Using Hash-based Contextual Signatures in Intrusion Detection," Computer Communications, vol. 38, pp. 50-59, 2014

[27] Y. Meng, W. Li, and L.F. Kwok, "Towards Adaptive Character Frequency-based Exclusive Signature Matching Scheme and its Applications in Distributed Intrusion Detection," Computer Networks, vol. 57, no. 17, pp. 3630-3640, 2013.

[28] W. Li, W. Meng, and L.F. Kwok, "An Evaluation of Single Character Frequency-Based Exclusive Signature Matching in Distinct IDS Environments," In: Proceedings of the 17th International Conference on Information Security (ISC), pp. 465476, 2014

[29] W. Meng, W. Li, and L.F. Kwok, "EFM: Enhancing the Performance of Signature-based Network Intrusion Detection Systems Using Enhanced Filter Mechanism," Computers $\mathcal{E}$ Security, vol. 43, pp. 189-204, 2014.

[30] W. Meng, W. Li, and L.F. Kwok, "Design of Intelligent KNNbased Alarm Filter Using Knowledge-based Alert Verificatic. in Intrusion Detection," Security and Communication Network$s$ 8(18), pp. 3883-3895, 2015.

[31] W. Meng, W. Li, Y. Xiang, and K.K.R. Choo, "Bay, '9п Inference-based Detection Mechanism to Defend Medical Smartphone Networks Against Insider Attacks," Journal of Network and Computer Applications 78, pp. 162-169. ' 1 , .

[32] W. Meng, W. Li, and L.F. Kwok, "Towards Ef ctive Tru tbased Packet Filtering in Collaborative Netwe" Envirr 1ments," IEEE Transactions on Network and ervice in. " gement, vol. 14, no. 1, pp. 233-245, 2017

[33] W. Meng, E.W. Tischhauser, Q. Wang, Y. 'Van , and . Han, "When Intrusion Detection Meets Bloc' hain 'hr logy: A Review," IEEE Access, vol. 6, no. 1, r · 10179-10,38, IEEE, 2018.

[34] W. Meng, K.-K.R. Choo, S. Furnell. A.V. Vası „os, and C.W. Probst, "Towards Bayesian-based .rus Management for Insider Attacks in Healthcare Softw re-Df ned Networks," IEEE Transactions on Network and Ser, . Management, 2018.

[35] A. Mishra, B.B. Gupta, and ..C. Joshı, "A Comparative study of Distributed Denial of $S$ rvice Attacks, Intrusion Tolerance and mitigation Techniques, ' Ir Pror edings of the $2011 \mathrm{Eu}$ ropean Intelligence and $\mathrm{Secur}_{\iota}, \boldsymbol{I}_{\boldsymbol{n}}$, srmatics Conference, $\mathrm{pp}$. 286-289, 2011.

[36] S. Nakamoto, "Bitc in: A pec -to-peer electronic cash system," http://bitcc n.org/ itcoin.pdf, 2008.

[37] V. Paxson, "Bro · Jystem _ur Detecting Network Intruders in Real-Time," C mputer 1 . tworks, vol. 31, no. 23-24, pp. 24352463, 1999.

[38] Papadopoulos, C. I ind I, R., Mehringer, J., Hussain, A., and Govindar - rOSSACK: Coordinated Suppression of Simultaneous At. cks n: 1 roceedings of the 2003 DARPA Information Surviva ' cy Conference and Exposition (DISCEX), pp. 94-96, 2003

[39] Porras, P.A. and Neumann, P.G.: Emerald: Event Monitoring Enabling Responses to Anomalous Live Disturbances. In: Pro- ceedings of the 20th National Ir ormation Systems Security Conference, pp. 353-365, 1997.

[40] M. Roesch, "Snort: Lightwei,ht intru. ' $n$ detection for networks," In: Proceedings of ${ }^{\top}$ "ix Lisa Conference, pp. 229238, 1999.

[41] K. Scarfone and P. Mell, 'Tui e to Intrusion Detection and Prevention Systems (INPS),' ' 'ST Special Publication 80094, Feb 2007

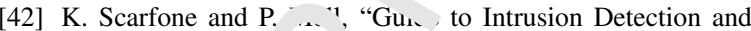
Prevention Syster , (ID _,", NIST Special Publication 80094, 2007.

[43] P.K. Sharma, S. Singh, '" -S. Jeong, and J.H. Park: "DistBlockNet: A Distr" uted Blockcilains-Based Secure SDN Architecture for IoT Jetworks, 'EEE Communications Magazine, vol. 55 , no. 9 , pp. ${ }^{78-85,2}, 7$.

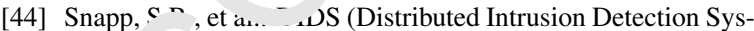
tem) - Motiv ?, Architecture, and An Early Prototype. In: Proce tir , of th : 14th National Computer Security Conferen ? pp. 167-1' o (1991)

[45] Snort. . n an open source network intrusion prevention and detection sys m (IDS/IPS). Homepage: http://www. snort. $\checkmark r /$

[4u, R. So. ._r and V. Paxson, "Outside the Closed World: On Usin $_{5}$ ' Machine Learning for Network Intrusion Detection," IEEE ryususium on Security and Privacy, pp. 305-316, 2010.

L4 " M. Steichen, S. Hommes, and R. State, "ChainGuard - A firew. 11 for blockchain applications using SDN with OpenFlow," $\imath$ : Proceedings of International Conference on Principles, Systems and Applications of IP Telecommunications (IPTComm), pp. 1-8, 2017.

[48] T.A. Tuan, "A Game-Theoretic Analysis of Trust Management in P2P Systems," Proceedings of ICCE, pp. 130-134, 2006.

[49] A. Valdes and D. Anderson, "Statistical Methods for Computer Usage Anomaly Detection Using NIDES," Technical Report, SRI International, January 1995.

[50] G. Vigna and R.A. Kemmerer, "NetSTAT: A Network-based Intrusion Detection Approach," Proc. Annual Computer Security Applications Conf. (ACSAC), pp. 25-34, 1998.

[51] L. Wang and Y. Liu, "Exploring Miner Evolution in Bitcoin Network," In: Mirkovic J, Liu Y, editors. Passive and Active Measurement. vol. 8995 of Lecture Notes in Computer Science, Springer, pp. 290-302, 2015.

[52] G. Wood, "Ethereum: A secure decentralised generalised transaction ledger," 2016, EIP-150 Revision.

[53] Y.-S. Wu, B. Foo, Y. Mei, and S. Bagchi, "Collaborative Intrusion Detection System (CIDS): A Framework for Accurate and Efficient IDS," In: Proceedings of the 2003 Annual Computer Security Applications Conference (ACSAC), pp. 234-244, 2003.

[54] K. Wüst and A. Gervais, "Do you need a blockchain?" IACR Cryptology ePrint Archive, vol. 2017, pp. 375, 2017. [Online]. Available: http://eprint.iacr.org/2017/375

[55] X. Xu et al., "The Blockchain as a Software Connector," In: Proceedings of the 13th Working IEEE/IFIP Conference on Software Architecture, pp. 1-10, 2016.

[56] Yegneswaran, V., Barford, P., and Jha, S.: Global Intrusion Detection in the DOMINO Overlay System. In: Proceedings of the 2004 Network and Distributed System Security Symposium (NDSS), pp. 1-17, (2004) 
Wenjuan Li is currently a Ph.D. student in the Department of Computer Science, City University of Hong Kong (CityU), and is holding an exchanged role at Technical University of Denmark (DTU), Denmark. Prior to this, she worked as a Research Assistant in CityU from 2013 to 2014, and was previously a Lecturer in the Department of Computer Science, $Z^{\prime}$ - a aing Foreign Language College, China. She was a Winner of Cyber Quiz and Computer Security Con petition, Final Round of Kaspersky Lab "Cyber Security for the Next Generation" Cor iere' ce in 2014. Her research interests include network management and security, collaborative $1 \mathrm{~h}$. TIsion detection, spam detection, trust computing, web technology and E-commerce technolog, She is a student member of IEEE.

Steven Tug is an exchanged student at Department of Applif 1 Mat' smatics and Computer Science, Technical University of Denmark (DTU), Denmark. He , 's a b jad interest in network and system security, like malware detection and smartphr le seririty. Before, he got some programming experiences from industry.

Weizhi Meng is currently an assistant professor in the 'apartm nt of Applied Mathematics and Computer Science, Technical University of Denmark (_TU), Denmark. He obtained his Ph.D. degree in Computer Science from the City Universi _ _.... Kung Kong (CityU), Hong Kong. He was known as Yuxin Meng and prior to joining DTU, he wu. ${ }^{1}$ ed as a research scientist in Infocomm Security (ICS) Department, Institute for Infoco $m$ kesearch, Singapore. His primary research interests include intrusion detection, smartph sec rity, biometric authentication, HCI security, blockchain security, trust management, and vuln rat. ity analysis. He is a member of IEEE.

Yu Wang received his Ph.D. degr e in omputer science from Deakin University, Victoria, Australia. He is currently an associaı ^rofe , or with the School of Computer Science, Guangzhou University, China. His research iter sts include network traffic analysis, mobile networks, social networks, and cyber security. 

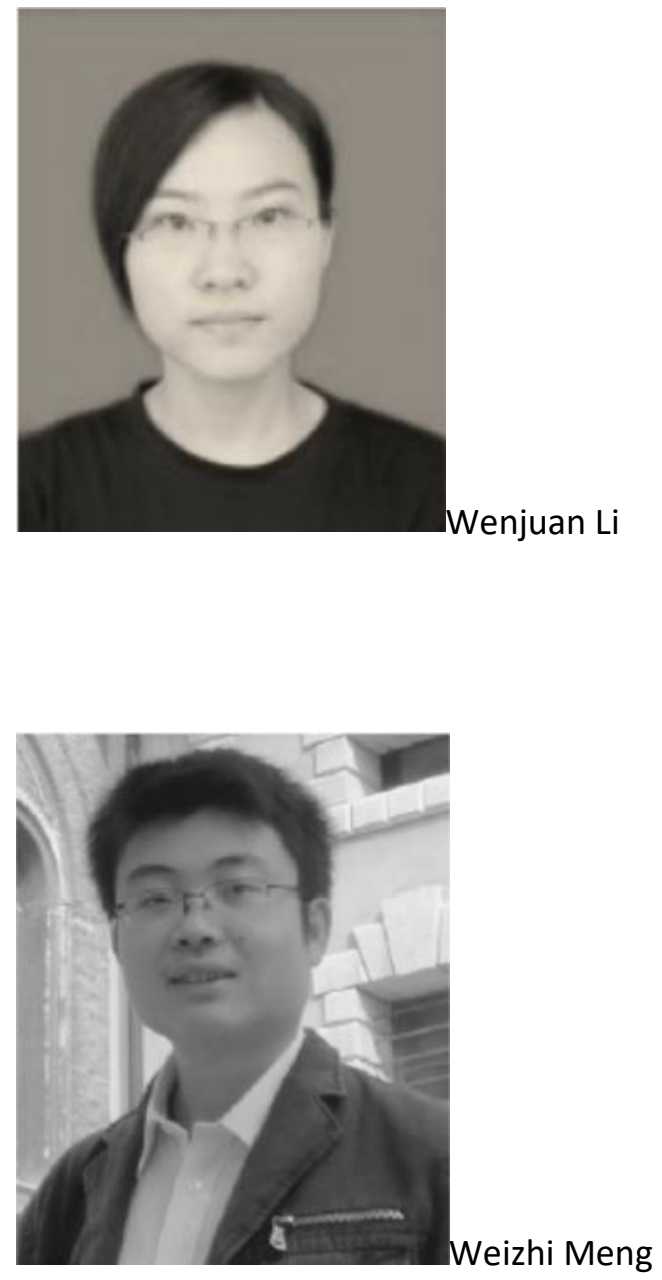

ru Wang 


\section{Highlights}

1. We propose CBSigIDS, a framework by combining blockchains with signatu - hased IDSs in a collaborative IoT environment.

2. Our framework enables various IDS nodes to incrementally produce and erif a signature (or rule) database without the need of a trusted intermediary.

3. We evaluated CBSigIDS in different environments and adversarial srenarı including both a simulated and a real CIDN environment.

4. We also compare and apply our approach into a blockchain-base. SDN application in a practical IoT environment. 